\title{
Distortion Cancellation by Polyphase Multipath Circuits
}

\author{
Eisse Mensink, Student Member, IEEE, Eric A. M. Klumperink, Member, IEEE, and Bram Nauta, Senior Member, IEEE
}

\begin{abstract}
It is well known that in balanced (or differential) circuits, all even harmonics are canceled. This cancellation is achieved by using two paths and exploiting phase differences of $180^{\circ}$ between the paths. The question addressed in this paper is: what distortion products (harmonics and intermodulation products) are canceled if more than two paths (and phases) are used? These circuits are called polyphase multipath circuits. It turns out that the more paths (and phases) are used, the more distortion products are canceled. Unfortunately, some intermodulation products cannot be canceled without also canceling the desired signal. An analysis of the impact of mismatch between the paths shows that the suppression of distortion products will be larger if more paths are used. As an application example, the design of an upconversion mixer with a clean output spectrum is presented.
\end{abstract}

Index Terms-Analog circuits, circuit analysis, distortion cancellation, harmonic rejection, image rejection, multipath, nonlinear circuits, polyphase.

\section{INTRODUCTION}

$\mathbf{N}$ ONLINEARITY, e.g., in RF amplifiers, filters and mixers, results in unwanted spectral components like harmonics and intermodulation products (distortion products). An overview of distortion mechanisms and analysis is given in a paper by Sansen [1]. As distortion products are usually unwanted, they should be removed or attenuated.

Linearization by feedback is possible but with limits. Especially in high frequency circuits, the amount of available loop gain is limited. Furthermore, feedback introduces instability risks.

Another commonly used method of removing distortion products is filtering. However, the achievable roll-of of filters is limited, impeding steep filtering. Moreover, the required filters are application specific, which is for instance a problem in realizing multi-standard radio transceiver systems.

This paper explores the use of polyphase multipath circuits [2] to cancel distortion products. Polyphase multipath circuits are circuits that use more than one path and exploit phase differences between these paths to cancel unwanted signals. As is well known that in balanced (or differential) circuits all even harmonics are canceled by using two paths and two phases $\left(0^{\circ}\right.$ and $180^{\circ}$ ), one might wonder which distortion products can be canceled with circuits that use more paths and phases.

In [2] it is shown that the more paths (and phases) are used, the more distortion products are canceled. It also turns out that some

Manuscript received November 1, 2004; revised February 2, 2005. This paper was recommended by I. M. Filanovsky.

The authors are with the University of Twente, 7500 AE Enschede, The Netherlands (e-mail: e.mensink@utwente.nl).

Digital Object Identifier 10.1109/TCSI.2005.852020 of the intermodulation products that are often a big problem in RF receivers, cannot be canceled without also canceling the desired signal. However, harmonics and most intermodulation products can be canceled. This could prove useful in designing multi-standard radio transmitters. By legislation, it is forbidden to transmit spectral components that fall outside the available frequency band for a certain standard, above a certain level. If filters are used, a different filter is needed for every standard. In contrast to filters, the polyphase multipath technique is very flexible. The same circuit can be used for all standards, while the harmonics are still canceled.

In Section II, the theory of polyphase multipath circuits is treated. After that, in Section III, the impact of mismatch between the paths is analyzed. The analysis shows that the more paths are used, the larger the suppression of the distortion products becomes. It also shows that the higher the harmonics that are to be suppressed, the more sensitive the suppression is to phase errors between the paths. In addition, this paper shows the design of an upconversion mixer with a clean output spectrum. This application example is simulated at transistor level (Section IV). The conclusions are given in Section V.

\section{Polyphase Multipath Technique}

\section{A. Distortion Products}

As mentioned in the introduction, unwanted spectral components often result from nonlinearity, e.g., in RF amplifiers, filters and mixers [1]. If $x(t)=A_{1} \cos \left(\omega_{1} t\right)+A_{2} \cos \left(\omega_{2} t\right)$ is the input of a memoryless nonlinear circuit, the output will not only consist of cosines with a fundamental frequency $\left(\omega_{1}\right.$ or $\left.\omega_{2}\right)$, but also of cosines with other frequencies: harmonics $\left(n \omega_{1}\right.$ or $\left.n \omega_{2}\right)$ and intermodulation products $\left(k \omega_{1}+m \omega_{2}\right)$ [Fig. 1(a) and 1(b)].

The question that we address in this paper is: can we cancel the harmonics and intermodulation products with the help of a polyphase multipath technique [Fig. 1(c)]?

\section{B. Polyphase Multipath Technique}

Fig. 2 shows a polyphase multipath circuit. The basic idea is to split an input signal into $N$ paths that consist of a phase shifter, a nonlinear circuit with distortion to be canceled, and another phase shifter. The nonlinear circuits are identical, but the phase shifts of the phase shifters differ for every path. At the end, the outputs of the different paths $\left(s_{1}, s_{2}, \ldots, s_{N}\right)$ are added to get the output signal.

The aim of the circuit of Fig. 2 is twofold. On the one hand, the desired signals should have equal phases at the end of every path [Fig. 3(a)]. In this way, they add constructively. On the 


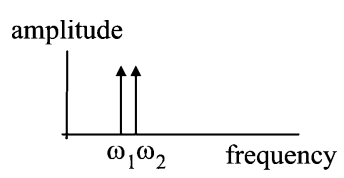

(a)

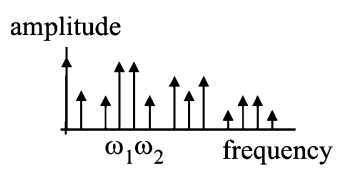

(b)

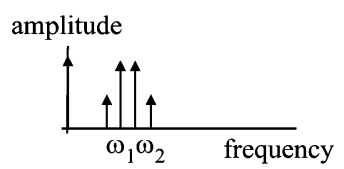

(c)

Fig. 1. (a) Input spectrum. (b) Output spectrum of a nonlinear circuit. (c) Output spectrum of a polyphase multipath circuit.

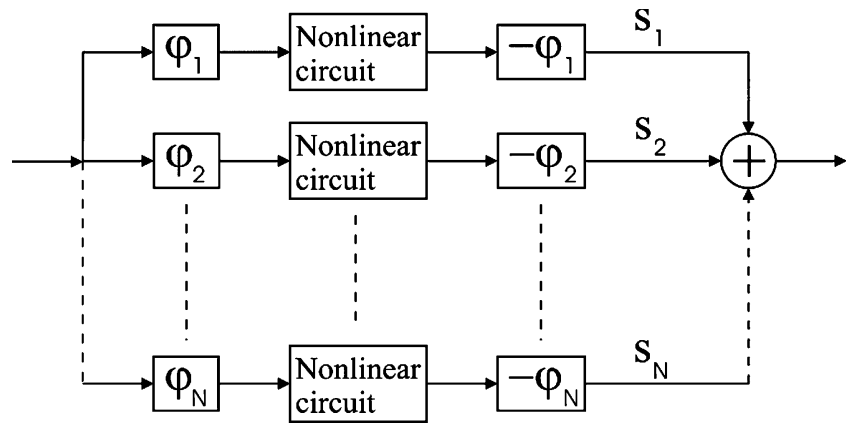

Fig. 2. Polyphase multipath circuit.

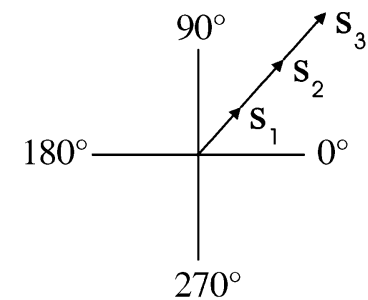

(a)

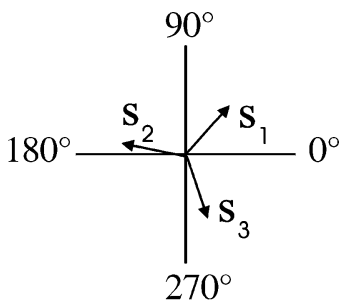

(b)
Fig. 3. (a) Phases at the output of the desired signals add constructively. (b) Phases at the output of the undesired signals cancel each other $(N=3)$.

other hand, the undesired signals (distortion products) should have a different phase at the end of every path and the phase differences between the paths should be chosen in such a way that the unwanted signals are canceled [Fig. 3(b)].

How can this situation be achieved? We can find this out by looking at the phases of the signals in the different paths. First, we will analyze the harmonics of the input signal, where the first harmonic is the desired signal. Intermodulation products will be considered afterwards.

\section{Phases of Harmonics}

If we combine the phase shifts of the phase shifters in the different paths into a vector $\varphi=\left[\varphi_{1}, \varphi_{2}, \ldots, \varphi_{N}\right]$, then the phases of the signals after the first phase shifters are $\underline{\varphi}$ (assuming $\varphi_{\text {in }}=0$ ). The nonlinear circuits generate harmonics of these signals.

We will assume that the circuits are weakly nonlinear and memoryless. Their behavior can be described by a Taylor approximation

$$
y(t)=a_{0}+a_{1} x(t)+a_{2} x^{2}(t)+a_{3} x^{3}(t)+\ldots
$$

where $x(t)$ is the input of the nonlinear circuit, $y(t)$ is the output and $a_{i}$ are constants. If $x(t)=A \cos \left(\omega t+\varphi_{i}\right)$, then the desired output of the nonlinear circuit will be $a_{1} A \cos \left(\omega t+\varphi_{i}\right)$ due to the $a_{1} x(t)$ term of (1). The other terms in this equation produce the distortion products. $a_{2} x^{2}(t)$ will give

$$
\begin{aligned}
a_{2} x^{2}(t) & =a_{2}\left(A \cos \left(\omega t+\varphi_{i}\right)\right)^{2} \\
& =\frac{1}{2} a_{2} A^{2}\left(1+\cos \left(2 \omega t+2 \varphi_{i}\right)\right) .
\end{aligned}
$$

In the same way, $a_{3} x^{3}(t)$ gives

$$
\begin{aligned}
a_{3} x^{3}(t) & =a_{3}\left(A \cos \left(\omega t+\varphi_{i}\right)\right)^{3} \\
& =\frac{1}{4} a_{3} A^{3}\left(3 \cos \left(\omega t+\varphi_{i}\right)+\cos \left(3 \omega t+3 \varphi_{i}\right)\right) .
\end{aligned}
$$

From these two equations, it is already seen that the phase of the input signal undergoes exactly the same operations as the frequency: if a term with $n \omega t$ is produced, then it will have a phase of $n \varphi_{i}$. In general, the phases of the $n$th harmonic after the nonlinear circuits (in vector notation) will be

$$
\underline{\varphi}_{\text {nonlinear }, n}=n \cdot \underline{\varphi} .
$$

The phase shifters after the nonlinear circuits set the phase of the desired signal $(n=1)$ back to zero. The higher harmonics undergo the same phase shifts (the phase shifters are assumed to be frequency independent). Therefore, the phases of the $n$th harmonic just before the adder will be

$$
\underline{\varphi}_{s, n}=(n-1) \cdot \underline{\varphi} .
$$

The desired signals $(n=1)$ will have the same phase in every path $\left(\underline{\varphi}_{s, n}=\underline{0}\right)$ and add constructively. The higher harmonics will have a different phase in every path $\left(\underline{\varphi}_{s, n} \neq \underline{0}\right)$ and can be canceled. It depends on the choice of $\varphi$ and the number of paths $N$ whether these harmonics are canceled or not.

\section{Canceling of Harmonics}

If the phases between the paths are chosen equidistant, thus $\underline{\varphi}=\left[0 * 360^{\circ} / N, 1 * 360^{\circ} / N, \ldots,(N-1) * 360^{\circ} / N\right]$, then (5) can be rewritten as

$$
\underline{\varphi}_{s, n}=\frac{(n-1)}{N} \cdot\left[0 \cdot 360^{\circ}, 1 \cdot 360^{\circ}, \ldots,(N-1) \cdot 360^{\circ}\right] \text {. }
$$

This equation shows that whenever $n=p * N+1(p=$ $0,1,2, \ldots)$, the phase in every path will be a multiple of $360^{\circ}$ leading to constructive addition [Fig. 3(a)]. It can be shown that in all other cases, so $n \neq p * N+1$, the phases are distributed equidistantly over $360^{\circ}$, leading to cancellation [Fig. 3(b)]. Therefore, for $N$ paths all harmonics are canceled, except the $(p * N+1)$ th harmonics. For example, for $N=2$ only the $(p * 2+1)$ th harmonics are not canceled, which are all uneven harmonics. So, all even harmonics are canceled, as known for balanced circuits. For $N=3$, the 4 th, 7 th, 10th, etc. harmonics 
TABLE I

Phase of $n$th Harmonic Just For AdDition in a Polyphase Multipath Circuit

\begin{tabular}{c|c|c|c|c|c|c|c|c|c}
\hline \hline $\mathbf{n}$ & $\mathbf{0}$ & $\mathbf{1}$ & $\mathbf{2}$ & $\mathbf{3}$ & $\mathbf{4}$ & $\mathbf{5}$ & $\mathbf{6}$ & $\mathbf{7}$ & $\mathbf{8}$ \\
\hline Phase of $\underline{\mathbf{s}}$ & $\underline{0}$ & $\underline{0}$ & $\underline{\varphi}$ & $2 \varphi$ & $3 \varphi$ & $4 \varphi$ & $5 \varphi$ & $6 \varphi$ & $7 \varphi$ \\
\hline \hline
\end{tabular}

TABLE II

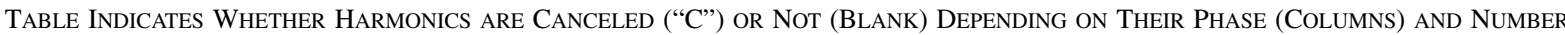
OF PATHS (ROWS), $\varphi=\left[0 * 360^{\circ} / N, 1 * 360^{\circ} / N, \ldots,(N-1) * 360^{\circ} / N\right]$

\begin{tabular}{|c|c|c|c|c|c|c|c|c|}
\hline $\begin{array}{l}\text { Phase of } \underline{\mathbf{s}} \\
\mathbf{N}\end{array}$ & $\underline{\mathbf{0}}$ & $\pm \Phi$ & $\pm 2 \Phi$ & $\pm \mathbf{3} \Phi$ & $\pm 4 \Phi$ & $\pm 5 \Phi$ & $\pm 6 \Phi$ & $\pm 7 \Phi$ \\
\hline 2 & & $\mathrm{C}$ & & $\mathrm{C}$ & & $\mathrm{C}$ & & $\mathrm{C}$ \\
\hline 3 & & $\mathrm{C}$ & $\mathrm{C}$ & & $\mathrm{C}$ & $\mathrm{C}$ & & $\mathrm{C}$ \\
\hline 4 & & $\mathrm{C}$ & $\mathrm{C}$ & $\mathrm{C}$ & & $\mathrm{C}$ & $\mathrm{C}$ & $\mathrm{C}$ \\
\hline 5 & & $\mathrm{C}$ & $\mathrm{C}$ & $\mathrm{C}$ & $\mathrm{C}$ & & $\mathrm{C}$ & $\mathrm{C}$ \\
\hline
\end{tabular}

are not canceled. In general, the more paths (and phases) are used, the more harmonics are canceled.

The same conclusion can be drawn from Tables I and II. Table I shows the phase of a certain harmonic [Table I is produced with the help of (5)]. Then, Table II can be used to see if a harmonic with this phase is canceled or not by a certain number of paths. The table, which is only valid for equidistant phases, shows that only if this phase $\left(\underline{\varphi}_{s, n}\right)$ is equal to $p * N * \underline{\varphi}$ the harmonic is not canceled. Combining this with (5) leads to the result that only the $(p * N+1)$ th harmonics are not canceled.

\section{E. Canceling of Intermodulation Products}

The canceling of intermodulation products can be analyzed in the same way as the canceling of the harmonics. Similar to (2) to (5), it can be shown that intermodulation products at $k \omega_{1}+m \omega_{2}$ ( $k$ and $m$ can be positive and negative) will have a phase of $(k+m) * \varphi$ after the nonlinear circuits and a phase of $(k+m-$ $1) * \varphi$ before the adder. The only difference with (5) is that $n$ is replaced by $k+m$. Therefore, if the $n$th harmonic is canceled, also the intermodulation products at $k \omega_{1}+m \omega_{2}$ with $k+m=n$ will be canceled.

Comparable to the results of Sections II-C and D, the only intermodulation products that are not canceled are the intermodulation products with $k+m=p * N+1$. Unfortunately, this means that the intermodulation products that have $k+m=1$ can never be canceled, because otherwise the desired signal $(n=1)$ would also be canceled. The most important intermodulation products that are in this category are third-order intermodulation products at $2 \omega_{1}-\omega_{2}$ and $2 \omega_{2}-\omega_{1}$. Unfortunately, these products are often a big problem in RF receivers. However, other harmonics and intermodulation products can be canceled.

Because the intermodulation products with $k+m=n$ will be canceled whenever the $n$th harmonic is canceled, Tables I and II can also be used for intermodulation products: just replace $\mathrm{n}$ by $k+m$. In the remainder of this paper, for briefness, we will only discuss the canceling of harmonics.

\section{F. Simulation Results}

Fig. 4 shows the simulation result of a two-tone test. In Matlab, (ideal) phase shifters and nonlinear circuits were implemented and the circuit of Fig. 2 was built for different numbers of paths. The fast Fourier transform (FFT) of the
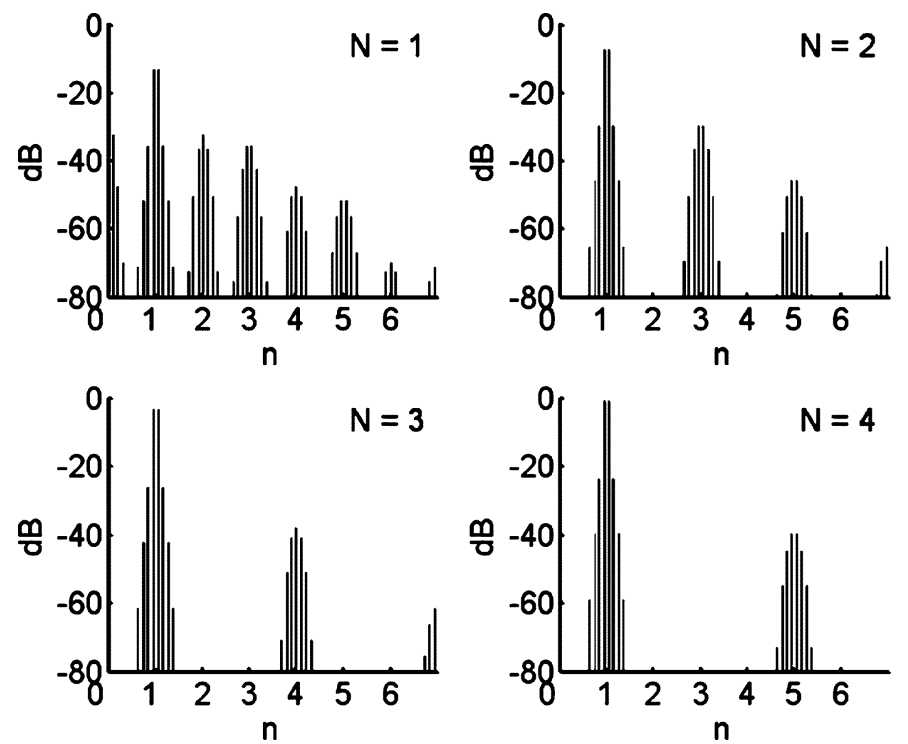

Fig. 4. Simulated output spectra of polyphase multipath circuits with one, two, three and four paths (two-tone test).

output of the circuits was calculated. On the horizontal axis is the number of the harmonics $n$. On the $y$ axis is the amplitude of the frequency components in decibels. The figure shows that the more paths are used, the more harmonics and intermodulation products are canceled. For $N$ paths, the $(p * N+1)$ th harmonics are the only components that are not canceled. For example, for $N=3$, the fourth and seventh harmonic are not canceled.

Because the frequencies $\left(\omega_{1}\right.$ and $\left.\omega_{2}\right)$ of the two tones are not far apart, the intermodulation products at $k \omega_{1}+m \omega_{2}$ with $k+m=n$ appear close to n. Fig. 4 shows that when the $n$th harmonic is canceled, the intermodulation products with $k+$ $m=n$ are also canceled.

\section{G. Mixer as a Wide-band Phase Shifter}

All phase shifters in Fig. 2 need to have a constant phase shift over all frequencies of interest. However, due to harmonics, the band of frequencies of interest is much wider after the nonlinear circuits. So the phase shifters after the nonlinear circuits have to have a constant phase shift over a much wider band of frequencies than the phase shifters before the nonlinear circuits. 
TABLE III

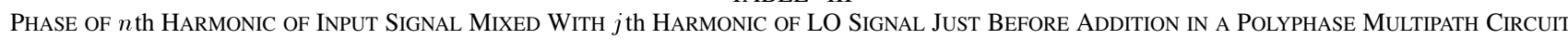
With MiXERS AS SECOND PHASE SHIFTERS

\begin{tabular}{l|c|c|c|c|c|c|c|c|c|c|c}
\hline \hline $\mathbf{n}$ & $-\mathbf{5}$ & -4 & -3 & -2 & -1 & $\mathbf{0}$ & $\mathbf{1}$ & $\mathbf{2}$ & $\mathbf{3}$ & $\mathbf{4}$ & $\mathbf{5}$ \\
\hline $\mathbf{0}$ & & & & & & & & & & & \\
\hline $\mathbf{1}$ & $6 \varphi$ & $4 \varphi$ & $3 \varphi$ & $2 \varphi$ & $\varphi$ & $\underline{0}$ & $\varphi$ & $2 \varphi$ & $3 \varphi$ & $4 \varphi$ & $5 \varphi$ \\
\hline $\mathbf{2}$ & $7 \varphi$ & $6 \varphi$ & $5 \varphi$ & $4 \varphi$ & $3 \varphi$ & $-2 \varphi$ & $-\varphi$ & $\underline{0}$ & $\varphi$ & $2 \varphi$ & $3 \varphi$ \\
\hline $\mathbf{3}$ & $8 \varphi$ & $7 \varphi$ & $6 \varphi$ & $5 \varphi$ & $4 \varphi$ & $-3 \varphi$ & $-2 \varphi$ & $-\varphi$ & $\underline{0}$ & $\underline{\varphi}$ & $2 \varphi$ \\
\hline $\mathbf{4}$ & $9 \varphi$ & $8 \varphi$ & $7 \varphi$ & $6 \varphi$ & $5 \varphi$ & $-4 \varphi$ & $-3 \varphi$ & $-2 \varphi$ & $-\varphi$ & $\underline{0}$ & $\varphi$ \\
\hline $\mathbf{5}$ & $10 \varphi$ & $9 \varphi$ & $8 \varphi$ & $7 \varphi$ & $6 \varphi$ & $5 \varphi$ & $-4 \varphi$ & $-3 \varphi$ & $-2 \varphi$ & $-\underline{\varphi}$ & $\underline{0}$ \\
\hline \hline
\end{tabular}

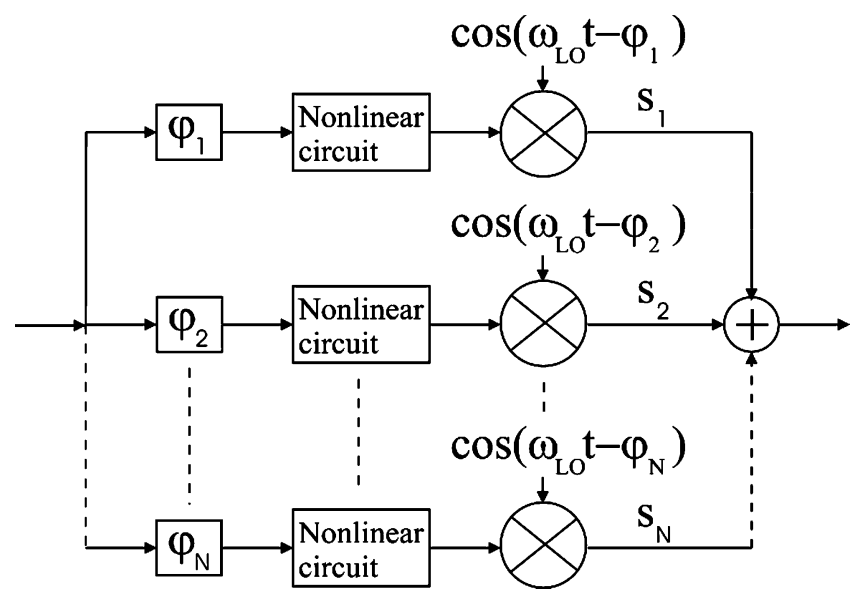

Fig. 5. Polyphase multipath circuit with mixers as wide-band phase shifters.

Phase shifters are often implemented using $R L C$ filters. However, it is difficult to realize a predefined phase shift over a wide band of frequencies with this type of filters. Also, polyphase filters can be used to generate phase shifted signals [3], but again, it is difficult to maintain a constant phase shift over a wide band of frequencies.

A very wide-band phase shifter can in principle be implemented with a mixer [4]. Since the phase shifters after the nonlinear circuits have to have a constant phase shift over a wide band of frequencies, these phase shifters are replaced by mixers. The input signal is assumed to have a small bandwidth; therefore, the phase shifters before the nonlinear circuits can be made with either $R L C$ filters, polyphase filters or, for base-band input signals, in the digital domain.

Fig. 5 shows the system with mixers. The mixers in the figure are assumed to be ideal mixers (mixer nonlinearity is modeled in the nonlinear circuits). For the frequency band in which the mixer works as a memoryless multiplier, the phase of the local oscillator (LO) signal is added to the phase of the input signal of the mixer, thus introducing a phase shift.

However, the mixer will introduce not only a phase shift, but also a frequency shift. So this technique can only be used if this frequency shift is desired (e.g., in an upconverter).

Furthermore, extra spectral components are introduced: due to sum and difference products of the input signal with the LO signal, both an upper and a lower sideband will appear. So in the frequency spectrum, harmonics will appear at both sides of the LO frequency.
Another problem that can appear in the mixer, is the presence of harmonics of the LO signal (for instance in a switching mixer). These harmonics of the LO signal will also be mixed with the input signal of the mixer and give even more extra spectral components. In the frequency spectrum, the harmonics of the input signal will also appear at both sides of the harmonics of the LO signal.

Assume now, that the desired signal is in the upper sideband, while a positive $\mathrm{n}$ means that the $n$th harmonic is in the upper sideband and a negative $\mathrm{n}$ means that the $n$th harmonic is in the lower sideband. ${ }^{1}$ (5) changes to

$$
\underline{\varphi}_{s, j, n}=\left\{\begin{array}{rr}
(n-j) \cdot \underline{\varphi}, & n \geq 0 \\
(-n+j) \cdot \underline{\varphi}, & n<0
\end{array}\right.
$$

for a spectral component at $j \omega_{\mathrm{LO}}+n \omega_{\mathrm{BB}}$ with $\omega_{\mathrm{BB}}$ the input frequency and $\omega_{\mathrm{LO}}$ the frequency of the LO signal. For (7), it is assumed that the LO signal can be modeled by a sinusoid that passes through a memoryless nonlinearity [see (1)]. This is, for instance, the case for a square wave.

Table III shows the result of (7) for common values of $n$ and $j$. Table III can be used in the same way as Table I: the phase of a certain spectral component at the output can be found in this table. Then, Table II can be used to see how many paths are needed to cancel that component. It turns out that most of the spectral components can be canceled if a sufficient number of paths is chosen. The only spectral components that the system of Fig. 5 does not cancel are the spectral components with $n=$ $j+p * N(p=\ldots,-2,-1,0,1,2, \ldots)$.

Unfortunately, for $p=0$, this formula becomes independent of N. So components with $n=j$ cannot be canceled with any number of paths. In Table III, these components have a phase $\underline{0}$.

\section{H. Simulation Results Mixer}

Fig. 6 shows a one-tone test in Matlab of a circuit with mixers with one path and Fig. 7 with seven paths. The spectra up to the eighth harmonic of the LO signal are plotted. The figures show that exactly those components remain at the output that were predicted by the theory: $3 \omega_{\mathrm{LO}}-4 \omega_{\mathrm{BB}}, 3 \omega_{\mathrm{LO}}+3 \omega_{\mathrm{BB}}, 5 \omega_{\mathrm{LO}}-$ $2 \omega_{\mathrm{BB}}$ and $7 \omega_{\mathrm{LO}}$ (compare with Table III, phases of $p * 7 * \varphi$ will $n o t$ be canceled with seven paths). Most of these components can be canceled by using a different number of paths.

\footnotetext{
${ }^{1}$ If the desired signal is in the lower sideband, the phases of the LO signals in Fig. 5 should be made positive instead of negative. Then, a positive $n$ means lower sideband and a negative $n$ means upper sideband.
} 


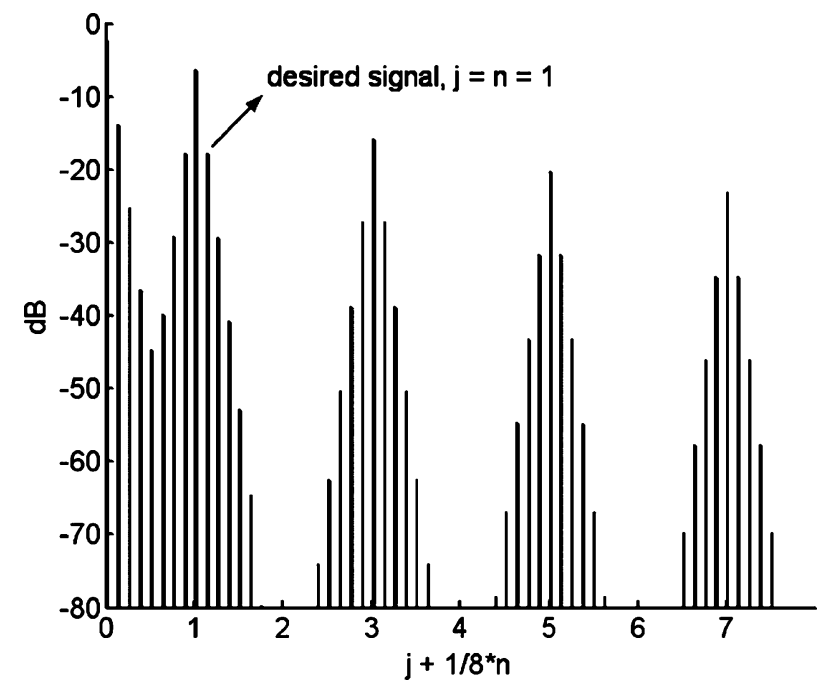

Fig. 6. Simulated output spectrum of a polyphase multipath circuit with mixers for $N=1$ (one path).

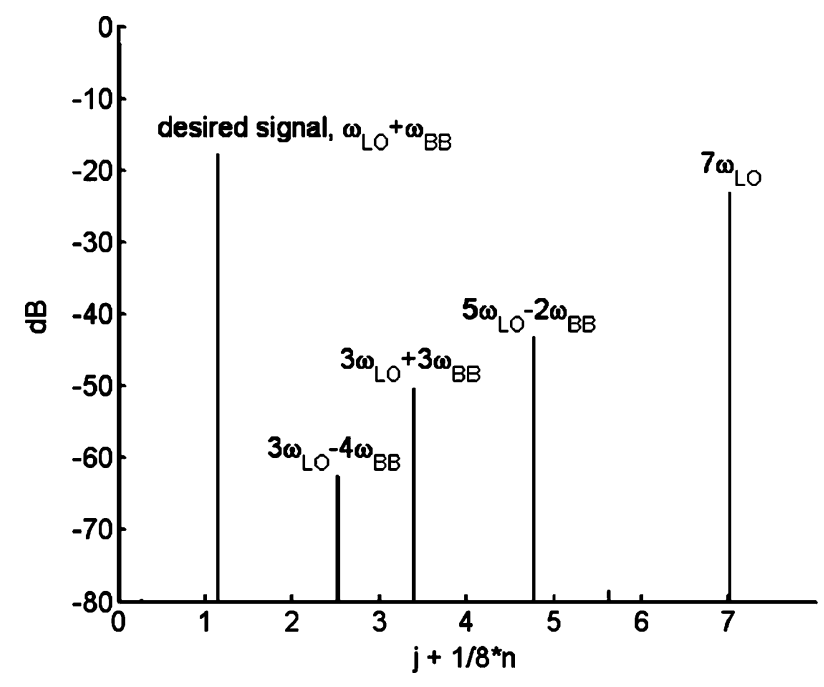

Fig. 7. Simulated output spectrum of a polyphase multipath circuit with mixers for $N=7$ (seven paths).

\section{Mismatch AND NOISE}

\section{A. Harmonic Rejection Ratio}

Of course, the canceling described in Section II depends on the matching between the paths. Imagine that in Fig. 5 the phase shifters have a phase error of $\underline{\theta}\left(\theta_{1}\right.$ in the upper path, $\theta_{2}$ in the second path, $\theta_{3}$ in the third path, etc.). Furthermore, imagine a gain mismatch of $\underline{\varepsilon}$ between the nonlinear circuits [described by (1)]. Finally, imagine a phase error of $\underline{\delta}$ between the LO signals. These errors are depicted in Fig. 8.

As a measure of the performance of the cancellation, the harmonic rejection ratio (HRR) for a certain distortion product $(j, n)$ is defined

$$
\mathrm{HRR}_{j, n}=\frac{P_{j, n, \text { reference }}}{P_{j, n, \text { rejected }}} .
$$

The first path of the circuit is used as a reference. First the output power of a certain distortion product is calculated if all

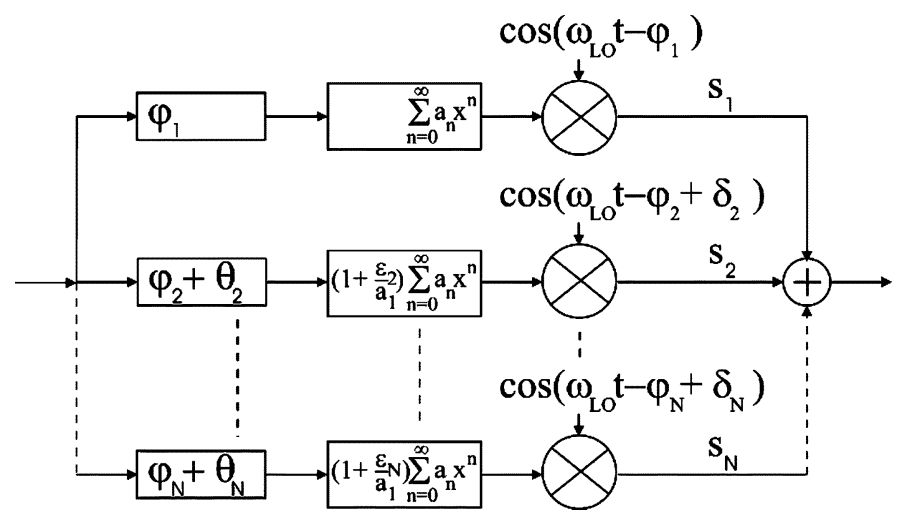

Fig. 8. Polyphase multipath circuit with mismatch.

$\mathrm{N}$ paths were identical to the first path (same phase shifts, no mismatches). This power is called $P_{j, n}$,reference. This power tells how strong the distortion product is at the output if the polyphase multipath technique is not used. After that, the output power of the distortion product is calculated with the polyphase multipath technique active. The phase shifts are chosen as in Section II. However, there is also mismatch between the paths. The resulting power, $P_{j, n}$,rejected , tells how strong the distortion product is at the output due to the mismatch. With no mismatch, this power would be zero, because the distortion product is canceled by the polyphase multipath technique. The ratio of the two powers tells how much the distortion product is suppressed by the circuit with mismatch compared to a circuit that does not use the polyphase multipath technique.

\section{B. Example: $\mathrm{HRR}_{1,3}$}

If we for instance look at a distortion product with $j=1$ and $n=3$ in a system with three paths, the HRR can be calculated as follows. As an input signal, $A \cos \left(\omega_{\mathrm{BB}} t\right)$ is chosen. The voltage of the distortion product at $\left(\omega_{\mathrm{LO}}+3 \omega_{\mathrm{BB}}\right)$ at the end of the first path is $1 / 8 a_{3} A^{3} \cos \left(3 \omega_{\mathrm{BB}} t+\omega_{\mathrm{LO}} t+2 \varphi_{1}\right)$. If all three paths are equal, the voltage at the output will be $3 / 8 a_{3} A^{3} \cos \left(3 \omega_{\mathrm{BB}} t+\right.$ $\left.\omega_{\mathrm{LO}} t+2 \varphi_{1}\right)$, so $P_{1,3, \text { reference }}=9 / 128 a_{3}^{2} A^{6}$.

In the circuit of Fig. 8 , the output power of $\omega_{\mathrm{LO}}+3 \omega_{\mathrm{BB}}$ can also be calculated. The voltage at the end of the first path is

$$
\frac{1}{8} a_{3} A^{3} \cos \left(3 \omega_{B B} t+\omega_{L O} t+2 \varphi_{1}\right)
$$

at the end of the second path

$$
\frac{1}{8}\left(a_{3}+\varepsilon_{2} \frac{a_{3}}{a_{1}}\right) A^{3} \cos \left(3 \omega_{B B} t+\omega_{L O} t+2 \varphi_{2}+3 \theta_{2}+\delta_{2}\right)
$$

and at the end of the third path

$$
\frac{1}{8}\left(a_{3}+\varepsilon_{3} \frac{a_{3}}{a_{1}}\right) A^{3} \cos \left(3 \omega_{B B} t+\omega_{L O} t+2 \varphi_{3}+3 \theta_{3}+\delta_{3}\right) .
$$

Adding these three signals gives (12), which is shown on the bottom of the next page. $\psi$ is not calculated, because we are only interested in the power of the signal.

In order to simplify the expression, the cosine terms in the amplitude are replaced by their Taylor approximations. The phases 
are given in radians. For three paths, $\varphi_{1}=0, \varphi_{2}=2 / 3 \pi$ and $\varphi 3=4 / 3 \pi \mathrm{rad}$

$$
\begin{aligned}
& \left.\cos (x)\right|_{x=\frac{2}{3} \pi} \approx-\frac{1}{2}-\frac{1}{2} \sqrt{3}\left(x-\frac{2}{3} \pi\right)+\frac{1}{4}\left(x-\frac{2}{3} \pi\right)^{2} \\
& \left.\cos (x)\right|_{x=\frac{4}{3} \pi} \approx-\frac{1}{2}+\frac{1}{2} \sqrt{3}\left(x-\frac{4}{3} \pi\right)+\frac{1}{4}\left(x-\frac{4}{3} \pi\right)^{2} .
\end{aligned}
$$

Furthermore, it is assumed that $\varepsilon_{i} \ll a_{i}$. The power of the $\omega_{\mathrm{LO}}+3 \omega_{\mathrm{BB}}$ product at the output is

$$
\begin{aligned}
P_{1,3, \text { rejected }}= & \frac{a_{3}^{2} A^{6}}{128}\left(\frac{\varepsilon_{2}^{2}}{a_{1}^{2}}+\frac{\varepsilon_{3}^{2}}{a_{1}^{2}}-\frac{\varepsilon_{2} \varepsilon_{3}}{a_{1}^{2}}+\left(3 \theta_{2}+\delta_{2}\right)^{2}\right. \\
& \left.+\left(3 \theta_{3}+\delta_{3}\right)^{2}-\left(3 \theta_{2}+\delta_{2}\right)\left(3 \theta_{3}+\delta_{3}\right)\right) .
\end{aligned}
$$

Now, the suppression can be calculated. The formula is given in (15) at the bottom of the page. The suppression depends on the square of the relative gain mismatches $\left(\left(\varepsilon_{i} / a_{1}\right)^{2}\right)$ and the square of the phase errors. The larger they are, the smaller the suppression, as shown in (15) at the bottom of the page .

Mismatch is a random process. Therefore, the $\varepsilon_{i}, \theta_{i}$ and $\delta_{j}$ can be seen as stochastic variables. We will assume that they are independent and all have a mean of zero. The cross products of the variables will be zero and can be removed from the equation. The expected value of the suppression of the third harmonic in a system with three paths will be

$$
E\left(\mathrm{HRR}_{1,3}\right)=\frac{P_{1,3, \text { reference }}}{P_{1,3, \text { rejected }}}=\frac{9}{2\left(\frac{\sigma_{\epsilon}^{2}}{a_{1}^{2}}+9 \sigma_{\theta}^{2}+\sigma_{\delta}^{2}\right)}
$$

where $\sigma_{x}^{2}$ is the variance of stochastic variable $x$ and $E()$ is the expectation operator.

\section{General Equation for $\mathrm{HRR}_{j, n}$}

More generally, the suppression of $j \omega_{\mathrm{LO}}+n \omega_{\mathrm{BB}}$ in a system with $N$ paths has been derived. The result is

$$
\begin{aligned}
E\left(\mathrm{HRR}_{j, n}\right) & =\frac{P_{j, n, \text { reference }}}{P_{j, n, \text { rejected }}} \\
& =\frac{N^{2}}{(N-1)\left(\frac{\sigma_{\varepsilon}^{2}}{a_{1}^{2}}+n^{2} \sigma_{\theta}^{2}+j^{2} \sigma_{\delta}^{2}\right)} .
\end{aligned}
$$

The more paths are used $(N)$, the larger the suppression becomes. Also, the higher the harmonic that is to be canceled ( $n$ and/or $j$ ), the less suppression is possible. However, in general the higher the harmonic, the weaker this signal is, so the less suppression is needed.

Equation (17) is also valid for intermodulation products. For intermodulation products at $k \omega_{1}+m \omega_{2}$, the $n$ in (17) should be replaced by $k+m$.

\section{Simulation Results}

The circuit of Fig. 8 was implemented in Matlab to calculate the influence of mismatch. The HRR of $\omega_{\mathrm{LO}}+3 \omega_{\mathrm{BB}}$ was calculated hundred times with random values for $\underline{\varepsilon}, \underline{\theta}, \underline{\delta}\left(\sigma_{\varepsilon}=0.03\right.$, $\left.\sigma_{\theta}=\sigma_{\delta}=0.017\right)$. Fig. 9 shows the result for $N=2$ to $N=8$ paths. A circle shows the expected value of $\mathrm{HRR}_{1,3}$ according to (17) and an $x$ shows the mean of the simulated values of $\mathrm{HRR}_{1,3}$. The theoretical and simulated values of $\mathrm{HRR}_{1,3}$ are almost equal. The simulations were repeated for other distortion products with the same result: theory and simulation results are almost the same, so (17) is a good estimate for the suppression of a certain distortion product.

\section{E. Power, Area, and Noise}

As explained in Section II, the desired signals in every path are in phase before they are added [Fig. 3(a)]. In this way, no signal power is lost due to the addition. This addition could for instance be done by summing the currents from every path.

$$
\begin{aligned}
& \left(\left(\frac{1}{8} a_{3} A^{3}\right)^{2}+\left(\frac{1}{8}\left(a_{3}+\varepsilon_{2} \frac{a_{3}}{a_{1}}\right) A^{3}\right)^{2}+\left(\frac{1}{8}\left(a_{3}+\varepsilon_{3} \frac{a_{3}}{a_{1}}\right) A^{3}\right)^{2}+2\left(\frac{1}{8} a_{3} A^{3}\right)\left(\frac{1}{8}\left(a_{3}+\varepsilon_{2} \frac{a_{3}}{a_{1}}\right) A^{3}\right)\right. \\
& \quad \times \cos \left(2 \varphi_{2}-2 \varphi_{1}+3 \theta_{2}+\delta_{2}\right)+2\left(\frac{1}{8} a_{3} A^{3}\right)\left(\frac{1}{8}\left(a_{3}+\varepsilon_{3} \frac{a_{3}}{a_{1}}\right) A^{3}\right) \cos \left(2 \varphi_{3}-2 \varphi_{1}+3 \theta_{3}+\delta_{3}\right) \\
& \left.\quad+2\left(\frac{1}{8}\left(a_{3}+\varepsilon_{2} \frac{a_{3}}{a_{1}}\right)\left(\frac{1}{8}\left(a_{3}+\varepsilon_{3} \frac{a_{3}}{a_{1}}\right) A^{3}\right) A^{3}\right) \cos \left(2 \varphi_{3}-2 \varphi_{2}+3 \theta_{3}-3 \theta_{2}+\delta_{3}-\delta_{2}\right)^{\frac{1}{2}} \cos \left(3 \omega_{B B} t+\omega_{L O} t+\psi\right)\right)
\end{aligned}
$$

$$
\begin{aligned}
\operatorname{HRR}_{1,3} & =\frac{P_{1,3, \text { reference }}}{P_{1,3, \text { rejected }}} \\
& =\frac{\frac{9 a_{3}^{2} A^{6}}{128}}{\frac{a_{3}^{2} A^{6}}{128}\left(\frac{\varepsilon_{2}^{2}}{a_{1}^{2}}+\frac{\varepsilon_{3}^{2}}{a_{1}^{2}}-\frac{\varepsilon_{2} \varepsilon_{3}}{a_{1}^{2}}+\left(3 \theta_{2}+\delta_{2}\right)^{2}+\left(3 \theta_{3}+\delta_{3}\right)^{2}-\left(3 \theta_{2}+\delta_{2}\right)\left(3 \theta_{3}+\delta_{3}\right)\right)} \\
& =\frac{9}{\frac{\varepsilon_{2}^{2}}{a_{1}^{2}}+\frac{\varepsilon_{3}^{2}}{a_{1}^{2}}-\frac{\varepsilon_{2} \varepsilon_{3}}{a_{1}^{2}}+\left(3 \theta_{2}+\delta_{2}\right)^{2}+\left(3 \theta_{3}+\delta_{3}\right)^{2}-\left(3 \theta_{2}+\delta_{2}\right)\left(3 \theta_{3}+\delta_{3}\right)}
\end{aligned}
$$




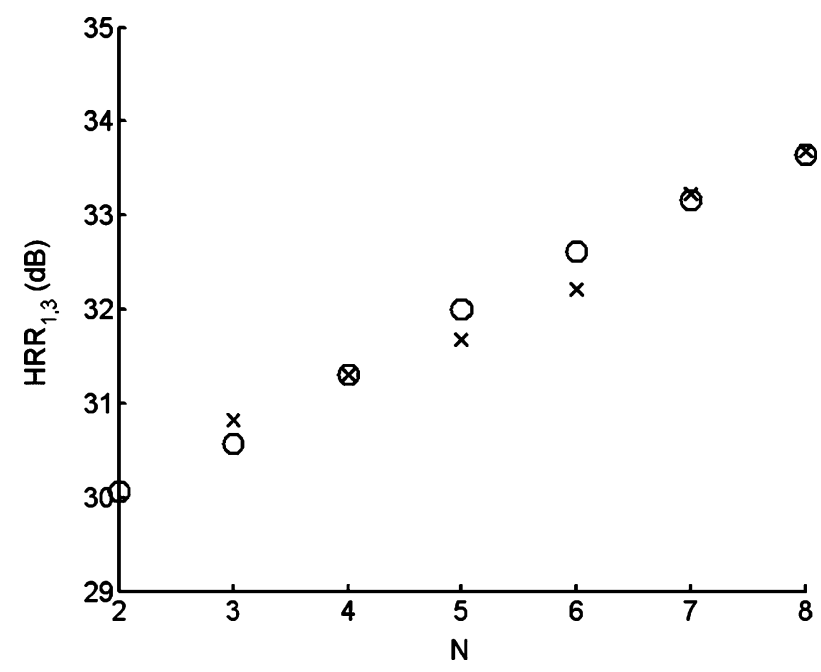

Fig. 9. Simulated $(x)$ and calculated (o) HRR for $\omega_{\mathrm{LO}}+3 \omega_{\mathrm{BB}}$ as a function of the number of paths.
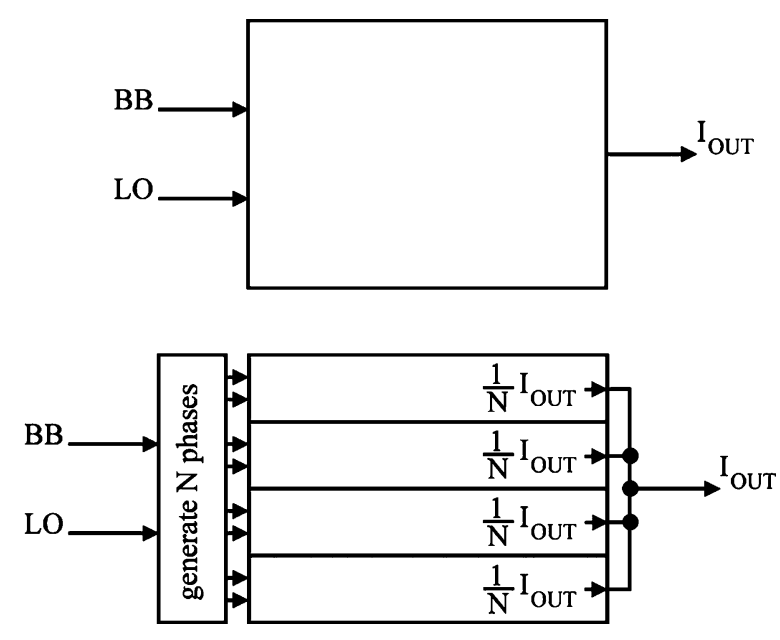

Fig. 10. Current per path in a system with $N$ paths is $N$ times smaller than the current in a system with one path.

Thus, for the same output power (and the same load impedance), the current per path in a system with $\mathrm{N}$ paths can be $N$ times smaller than the current in a system with one path [see Fig. 10].

The drain current of a transistor is proportional to its width. Therefore, also the area per path can be $N$ times smaller. So the total area for the BB to RF conversion remains the same. Only some extra area is needed to generate the phases. The generation of $N$ LO phases can for instance be done with a DLL.

If the current per path is $N$ times smaller, then in general the noise power per path can also be $N$ times smaller. Therefore, the total noise power after the addition is independent of $N$ and the signal-to-noise-ratio is not deteriorated by the polyphase multipath technique. However, the generation of the $N$ phases can introduce some extra noise.

\section{APPLICATION EXAMPLES}

This section shows some application examples of the technique described in the previous sections. It shows how existing

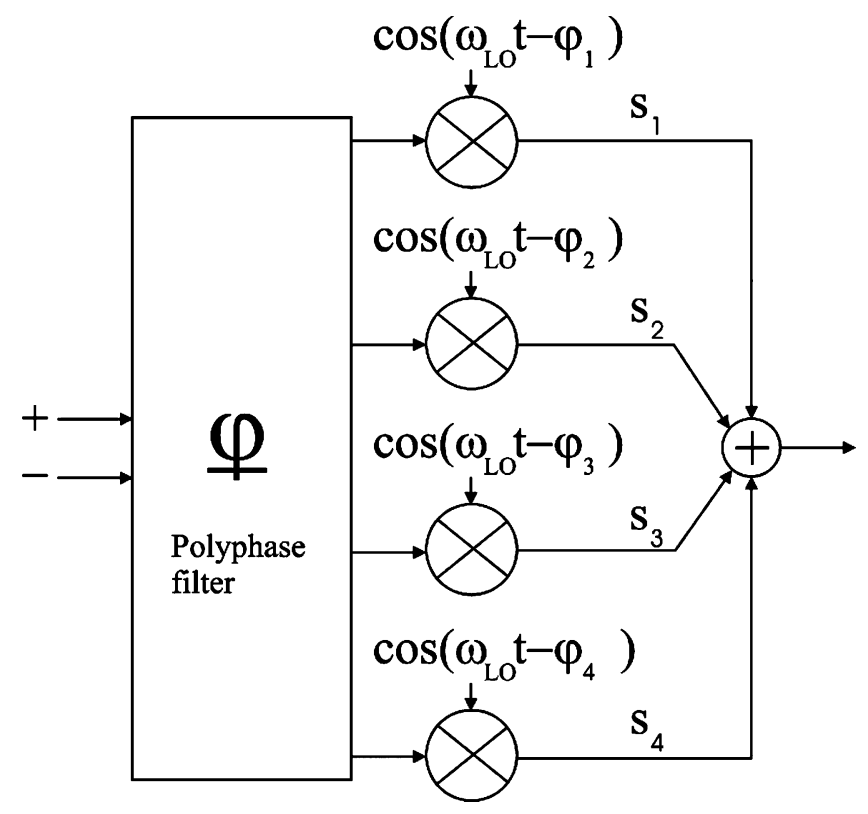

Fig. 11. Image reject structure of Gingell $(N=4)$.

circuits can be analyzed with the theory in Sections II and III and how new circuits can be generated.

\section{A. Balanced Circuits}

A well-known application is the use of balanced (or differential) circuits. In these circuits, $N=2$ and $\varphi=\left[0^{\circ}, 180^{\circ}\right]$. The nice thing about these circuits is that the implementation of the necessary phase shifts can be done very easily. Instead of using passive $R L C$ filter networks or mixers, the input signal is simply inverted. At the output, the difference is measured between the two outputs (combining the last phase shifter and the adder in Fig. 1). Apart from the simple implementation, these $180^{\circ}$ "phase shifts" are very wide-band (recall from Section II-F that this wide-band character of the phase shifters was necessary).

Table II shows that balanced circuits cancel those harmonics at the output that have a phase of $\underline{\varphi}, 3 \underline{\varphi}, 5 \underline{\varphi}$, etc. Comparing this with Table I gives the conclusion that balanced circuits cancel all the even harmonics, which is a well-known result.

\section{B. Generation of Clean $\mathrm{LO}$}

Another application can be the generation of a clean LO signal: the harmonics of the LO signal are to be canceled. This is for instance done in [5] and [6]. However, both techniques seem to have drawbacks. In [5], the third and seventh harmonic of the LO signal are canceled. However, they need linear mixers, which have for instance worse noise performance than switching mixers. In [6], the third and fifth harmonic of the LO signal are canceled. Three paths are used. However, the phases of the desired signal (fundamental of LO signal) are not equal at the end of the three paths. They are $45^{\circ}$ and $90^{\circ}$ out of phase. Therefore, there will be loss of desired signal when the signals in the three paths are added up. 


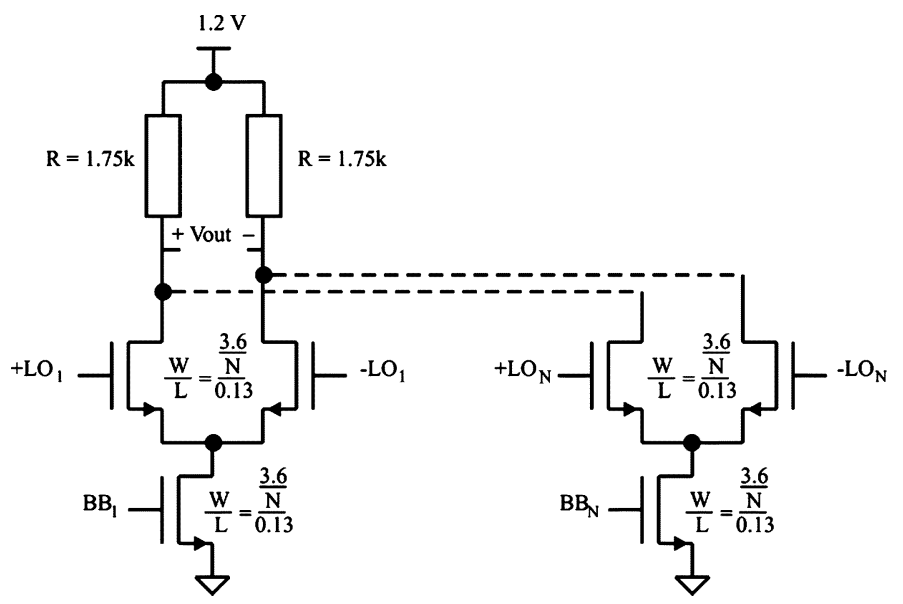

Fig. 12. Polyphase multipath mixer circuit. The bottom part is placed $\mathrm{N}$ times in parallel (N paths). The input signals $\mathrm{BB}_{1}$ to $\mathrm{BB}_{N}$ have phases of $\varphi_{1}$ to $\varphi_{N}$ and the differential LO signals $\mathrm{LO}_{1}$ to $\mathrm{LO}_{N}$ have phases of $-\varphi_{1}$ to $-\varphi_{N}$.

The use of polyphase multipath circuits also creates a clean LO signal. In [3], Gingell states that the image reject structure (see Fig. 11 for $N=4$ ) cancels all the harmonics of the LO signal up to and including the $(N-2)$ th harmonic. The polyphase filter in this figure performs the phase shifts of the input signal. The difference between Fig. 11 and Fig. 5 is the nonlinear circuits before the mixers. So the image reject structure of Gingell can be analyzed by looking at the columns of Table III with $n=-1$ and $n=1$. For $N=4$, Table II states that harmonics with phases of $\underline{\varphi}, 2 \underline{\varphi}$ and $3 \underline{\varphi}$ will be canceled and harmonics with a phase of $4 \underline{\varphi}$ will not be canceled. Looking at Table III, columns $n=-1$ and $n=1$, the first harmonic of the LO signal that is not canceled is the third harmonic $(j=3)$. This is exactly as stated by Gingell. However, Table III is more general, since it also looks at the harmonics of the input (baseband) signal.

\section{Upconversion Mixer With Clean Output Spectrum}

The previous application examples show how the theory of this paper can be used to analyze existing techniques. In this section, the polyphase multipath technique is used to generate a new circuit: an upconversion mixer with a clean output spectrum. Because of this clean output spectrum, no or less filtering is needed (see also [6]).

The mixer is simulated at transistor level to show that the technique also works with practical building blocks. The schematic is shown in Fig. 12. The circuit was first simulated with one path and after that with eight paths $\left(\underline{\varphi}=\left[0,45^{\circ}, 90^{\circ}, 135^{\circ}, 180^{\circ}, 225^{\circ}, 270^{\circ}, 315^{\circ}\right]\right)$ in a $0.13-\mu \mathrm{m}$ CMOS process. The results of both simulations are in Figs. 13 and 14. An FFT was performed on the differential output voltage (Vout) of the structure. As the figures show, the circuit with eight paths cancels almost all the distortion products generated by the nonlinearity of the transistors. Also, the higher harmonics of the LO signal are cancelled. As predicted [see (7)], the distortion products at $3 \omega_{\mathrm{LO}}+3 \omega_{\mathrm{BB}}, 5 \omega_{\mathrm{LO}}-3 \omega_{\mathrm{BB}}$, $7 \omega_{\mathrm{LO}}-\omega_{\mathrm{BB}}$ and $9 \omega_{\mathrm{LO}}+\omega_{\mathrm{BB}}$ are not canceled. A two-tone test (Fig. 15) of the circuit with eight paths shows that third order intermodulation products are not canceled, again as predicted.

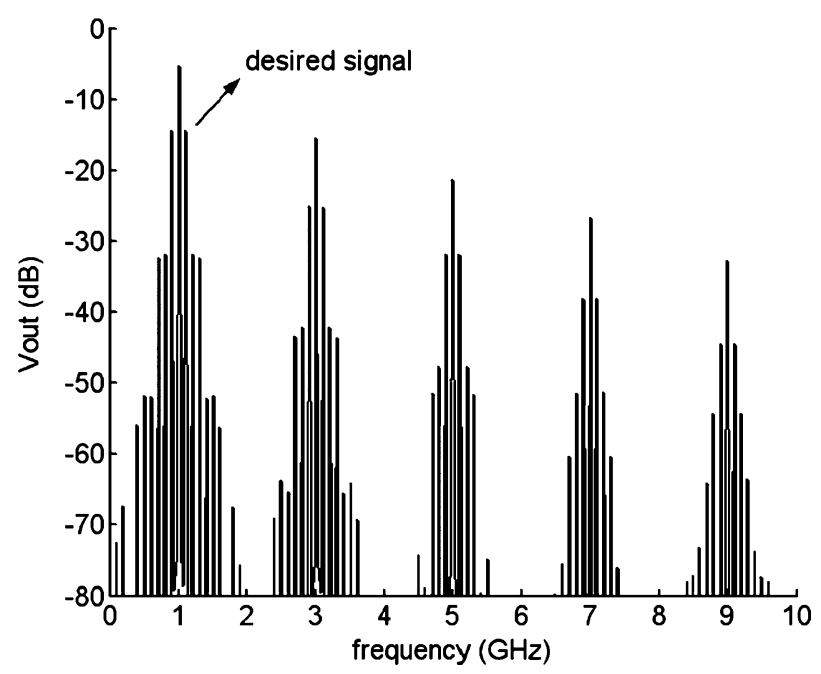

Fig. 13. Simulated output spectrum of mixer circuit (Fig. 11) with one path.

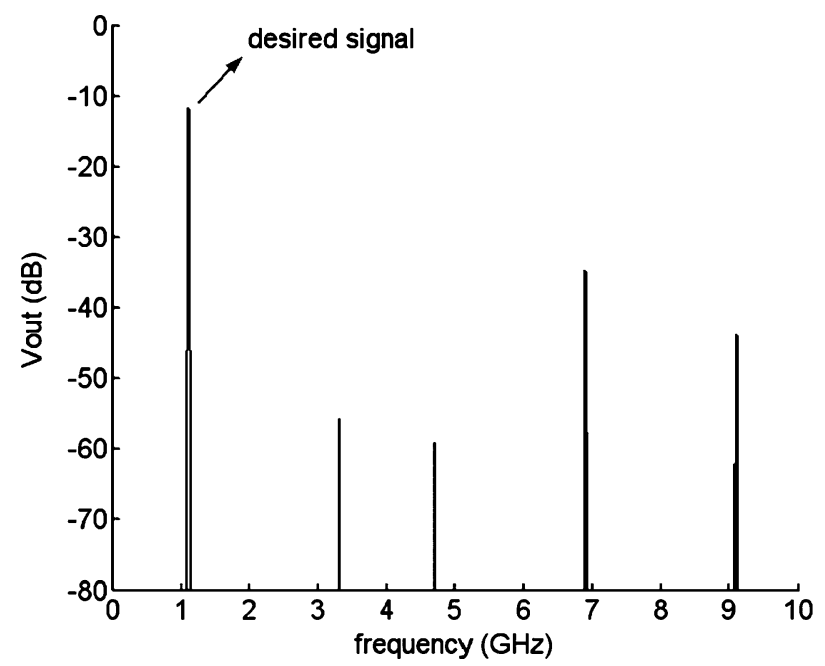

Fig. 14. Simulated output spectrum of mixer circuit (Fig. 11) with eight paths.

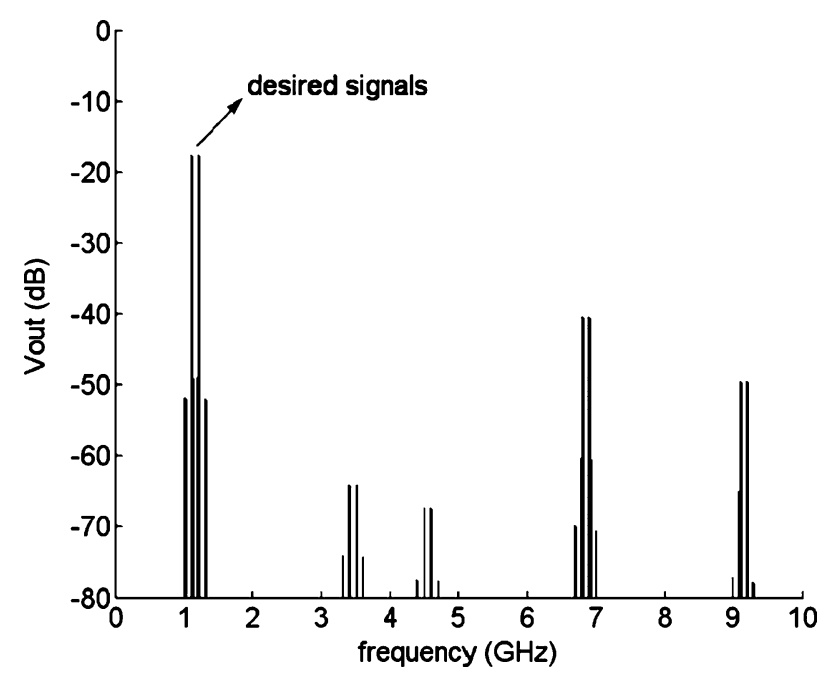

Fig. 15. Simulated output spectrum of mixer circuit (Fig. 11) with eight paths, two-tone test.

However, intermodulation products are mainly a problem in receivers where strong interferers are present in the input signal. They are less of a problem in an upconversion mixer. Fig. 15 


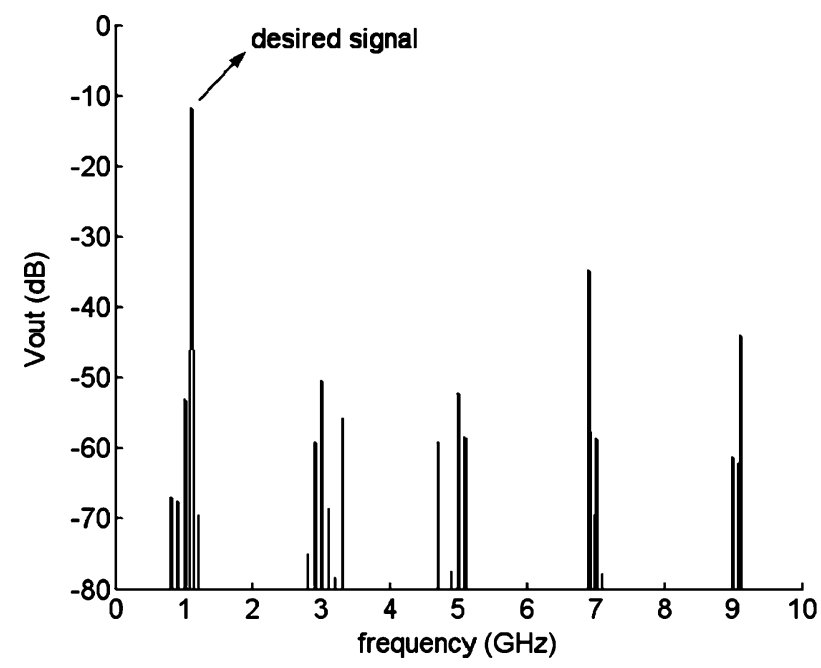

Fig. 16. Simulated output spectrum of mixer circuit (Fig. 11) with eight paths, with mismatch.

shows that the intermodulation products are $34 \mathrm{~dB}$ below the desired signals.

Finally, the circuit with eight paths was simulated with random mismatches between the transistors and random phase errors both between the input signals and between the LO signals. It was found that the mismatches between the transistors as simulated with the process statistics had a negligible impact on the suppression of the harmonics compared to phase mismatches [of about $1^{\circ}$ ]. In Fig. 16, the result for a circuit with phase mismatches is plotted. For $\theta_{1}, \theta_{2}, \ldots, \theta_{8}$ and $\delta_{1}, \delta_{2}, \ldots, \delta_{8}$ random numbers where generated with $\sigma_{\theta}^{2}=3.6 \cdot 10^{-4}$ and $\sigma_{\delta}^{2}=1.4 \cdot 10^{-4}$ (phase errors of about $1^{\circ}$ ). Only the result of one simulation is plotted, as the simulations take a long time. Comparing this figure with Fig. 13 learns that for instance the component at $3 \omega_{\mathrm{LO}}$ is suppressed by $35 \mathrm{~dB}$ and $5 \omega_{\mathrm{LO}}+\omega_{\mathrm{BB}}$ by $27 \mathrm{~dB}$.

This design shows a mixer that works at $1 \mathrm{GHz}$, while having a clean output spectrum up to $6.9 \mathrm{GHz}$ : the largest unwanted spectral component in this frequency range is $39 \mathrm{~dB}$ below the desired signal. This application example shows that by using the polyphase multipath technique no or less filtering is needed.

\section{CONCLUSION}

It has been shown that with polyphase multipath circuits it is very well possible to cancel distortion products produced by a nonlinear circuit. The more paths (and phases) are used, the more distortion products are canceled. In this paper tables are presented that can be used to determine whether a certain spectral component will be canceled or not, depending on the number of paths. Unfortunately, some intermodulation products (like third order intermodulation products at $2 \omega_{1}-\omega_{2}$ and $2 \omega_{2}-\omega_{1}$ ) cannot be canceled without also canceling the desired signal.
If a mixer is used as a phase shifter, extra spectral components appear: components in both an upper and a lower sideband around the harmonics of the LO signal. Most of these components can again be canceled by the polyphase multipath circuits if a sufficient number of paths is used. However, the components at $j \omega_{\mathrm{LO}}+n \omega_{\mathrm{BB}}$ where $n=j$ cannot.

Of course, the technique relies on matching between the paths. A formula ((17)) was derived that estimates the suppression of a certain harmonic if the variances of $\underline{\varepsilon}, \underline{\theta}, \underline{\delta}$ are known (see Fig. 8). The formula shows that the more paths are used, the larger the suppression. However, the suppression becomes less for higher harmonics.

With regard to power, area and noise, it can be concluded that for the same output power the use of more paths costs no extra area and introduces no extra noise. However, the generation of the phases gives some area overhead and could introduce some noise.

The theory of this paper is used to analyze existing circuits, but can also be used to generate new circuits. As an example, an upconversion mixer design at $1 \mathrm{GHz}$ was presented with a simulated clean output spectrum up to $6.9 \mathrm{GHz}$ (largest unwanted specral component is $39 \mathrm{dBc}$ ).

Both high-level simulations and transistor level simulations show that this technique can be used to design circuits with an enhanced spectral purity. Because no filters are needed, this technique is very flexible and could for instance be used in designing multi-standard radio transceiver systems.

\section{REFERENCES}

[1] W. Sansen, "Distortion in elementary transistor circuits," IEEE Trans. Circuits Syst. II, Analog Digit. Signal Process., vol. 46, no. 3, pp. 315-325, Mar. 1999.

[2] E. Mensink, E. A. M. Klumperink, and B. Nauta, "Distortion cancellation via polyphase multipath circuits," in Proc. 2004 IEEE Int. Symp. Circuits and Systems, May 2004.

[3] M. J. Gingell, "Single sideband modulation using sequence asymmetric polyphase networks," Elect. Commun., vol. 48, pp. 21-25, 1973.

[4] D. K. Weaver, "A third method of generation and detection of singlesideband signals," in Proc. Inst. Radio Eng., vol. 44, Dec. 1956, pp. 1703-1705.

[5] M. A. F. Borremans, C. R. C. De Ranter, and M. S. J. Steyeart, "A CMOS dual-channel, $100-\mathrm{MHz}$ to $1.1-\mathrm{GHz}$ transmitter for cable application," IEEE J. Solid-State Circuits, vol. 34, no. 12, pp. 1904-1913, Dec. 1999.

[6] J. A. Weldon et al., "A 1.75-GHz highly integrated narrow-band CMOS transmitter with harmonic-rejection mixers," IEEE J. Solid-State Circuits, vol. 36, no. 12, pp. 2003-2015, Dec. 2001.

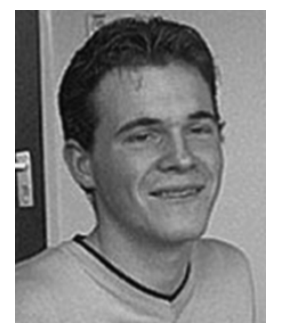

Eisse Mensink was born in 1979, in Almelo, The Netherlands. He received the M.Sc. degree in electrical engineering (cum laude) from the University of Twente, Enschede, The Netherlands, in 2003. He is currently working toward the Ph.D. degree at the same university on the subject of high-speed on-chip communication. 


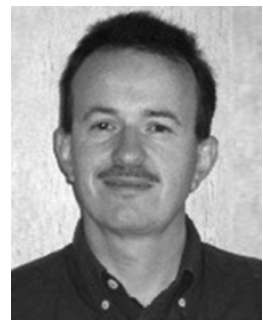

Eric A. M. Klumperink (M'98) was born in 1960, in Lichtenvoorde, The Netherlands. He received the B.Sc. degree from HTS, Enschede, The Netherlands, and the Ph.D. degree from the University of Twente, Enschede, The Netherlands, in 1982 and 1997, respectively.

After a short period in industry, he joined the Faculty of Electrical Engineering, University of Twente, in 1984, where he was mainly engaged in analog CMOS circuit design and research. This resulted in several publications and his Ph.D. thesis, in 1997, on the subject of "Transconductance Based CMOS Circuits." He is currently an Assistant Professor in the IC-Design Laboratory, University of Twente, where he is also involved in the MESA+ Research Institute. His research interest is in design issues of HF CMOS circuits, especially for front-ends of integrated CMOS transceivers. He holds four patents and has authored and co-authored more than 50 journal and conference papers.

Dr Klumperink is co-recipient of the ISSCC 2002 "Van Vessem Outstanding Paper Award."

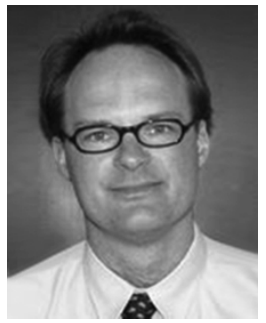

Bram Nauta (M'91-SM'03) was born in Hengelo, The Netherlands, in 1964. He received the M.Sc degree (cum laude) in electrical engineering from the University of Twente, Enschede, The Netherlands, and the Ph.D. degree from the same university on the subject of analog CMOS filters for very high frequencies, in 1987, and 1991, respectively.

In 1991, he joined the Mixed-Signal Circuits and Systems Department, Philips Research, Eindhoven, The Netherlands, where he worked on high-speed analog-digital converters. From 1994, he led a research group in the same department, working on "analog key modules." In 1998, he returned to the University of Twente, as Full Professor heading the IC Design group in the MESA+ Research Institute and Department of Electrical Engineering. His current research interest is analog CMOS circuits for transceivers. Besides, he is also Part-Time Consultant in industry and in 2001 he co-founded Chip Design Works.

His Ph.D. dissertation was published as a book: Analog CMOS Filters for Very High Frequencies (Boston, MA: Kluwer, 1993). He holds 11 patents in circuit design and he received the "Shell Study Tour Award" for his Ph.D. dissertation. From 1997-1999 he served as an Associate Editor of IEEE TRANSACTIONS ON CIRCUITS AND SySTEMS - II: ANALOG AND Digital Signal PROCESSING, and in 1998 he served as Guest Editor for IEEE JouRnAL OF SOLID-STATE CIRCUITS. In 2001 he became Associate Editor for IEEE JOURNAL OF SOLIDSTATE CIRCUITS and he is also member of the technical program committee of ESSCIRC and ISSCC. He is co-recipient of the ISSCC 2002 "Van Vessem Outstanding Paper Award." 